expanded local capacity and transformed the quality and experience of supportive and palliative care Some patients were identified as being not in need of specialist hospice beds or acute hospital admission but had non-complex requirements that could not be met in their ordinary home environment. A single bed within an Intermediate Care Unit was made available to support this care.

Methods Patients with non-complex needs are considered for admission to this bed if they are known to the MMSPCSNL and assessed by a band 7 clinician or doctor within the team at time of need. The bed is not suitable for individuals with complex needs or requiring daily medical review/input. During their admission they are supported by the staff within the unit as for any other inpatient in addition to regular review by the specialist palliative care team.

Results Since the pilot commenced, the skills and experience of the staff in the unit has increased with admission of patients with varying needs. The utilisation of the bed has been for multiple reasons from rehabilitation following cancer treatment in acute hospitals, to social crisis admissions, noncomplex end of life care and for combined reasons. During the first 12 months of the pilot 8 patients were supported with an admission length ranging from 12 to 65 days. 6 were discharged, 2 died in the compassionate bed as their preferred place of care.

Conclusion This pilot has achieved multiple positive outcomes such as appropriate care for patients in social crisis preventing acute hospital admissions, enhanced skills for unit staff and flexibility of approach with all teams involved. Funding is ongoing - the pilot has received favourable results and comments.

\section{CONSIDERING LIFE IMPACT OUTCOMES ON THE EARLY IDENTIFICATION OF PATIENTS IN NEED OF PALLIATIVE CARE ON THE DEVELOPMENT OF PREDICTIVE MODELS}

Mateos MJ Caballero, MS Giménez Campos, V Blanes-Selva, A Fernandes, JM GarcíaGiménez, A Duarte-Martinez, ME Gas López. Joint Research Unit in ICT applied to reengineering socio-sanitary process.Departament de Salut Valencia La Fe; Departament de Salut Valencia La Fe; Biomedical Data Science Lab ITACA Institute. Universitat Politecnica de Valencia; Santa Casa da Misericordi

\subsection{6/spcare-2020-PCC.136}

Introduction Stratification and predictive models have been proposed to support clinical decisions or to identify vulnerability-based sub-populations of complex patients. In particular, predicting mortality also has been one of the initiatives proposed on the prediction of palliative care (PC) needs. However, models considering life impact outcomes, such as negative effects due to illnesses, may be more able to contribute to identifying older patients who may benefit from early PC. The aim of this study was to review the current state of the art on health indicators which could suggest a declining trajectory of end-of-life in patients aged $>65$ with chronic disease(s).

Method A literature review was carried out in PubMed-Medline (February 20, 2019). Two research questions (RQ) were formulated (RQ1: mortality or survival prediction; RQ2: identification of patients with PC needs). Study selection was performed independently by two reviewers under the following inclusion criteria: subject sample aged $>65$, with chronic disease profile, published in English. Outcomes were categorised using the Core Outcome Measures in Effectiveness TrialsCOMET taxonomy, specifically the core area: Life Impact.

Results The keyword search gathered 712 papers for RQ1 and 696 for RQ2. After screening papers and eliminating duplicates, 101 studies were considered for retrieving information. We identified 1723 outcomes candidates but, after decoding abbreviations and concealing terms, only 484 remained. Of these, 83 terms were aligned with the core area Life Impact, being grouped under the following subdomains: cognitive $(n=13)$, physical $(n=28)$, social $(n=16)$ and emotional $(n=10)$ functioning; quality of life $(n=1)$; perceived health status $(n=6)$ and care delivered $(n=9)$.

Conclusion The literature review allowed us to collect updated life impact outcomes related to repercussions of illnesses which may guide the development of predictive modelling beyond a mortality approaching paradigm under the clinical setting mentioned.

\section{PATIENTS WITH PALLIATIVE CARE NEEDS NOT SO DISSIMILAR TO THOSE OF FRAILTY: TIME FOR SHARING APPROACHES}

Declan A Cawley, Jasmine Mann, Beverley Pryke. St Michael's Hospice, Medway NHS Foundation Trust, Wisdom Hospice

\subsection{6/spcare-2020-PCC.137}

Background Frailty within recent years has become a priority for healthcare providers looking at how anticipatory care plans can influence the potential and avoidable possibility of hospital admissions. Similarly, supportive and palliative care aims to meet individuals' preferences and wishes with hospital admission avoidance a high priority. The hospital setting is therefore an ideal opportunity when needs are identified to set in motion anticipatory/advance care plans, specifically looking at admission avoidance.

Aim To explore the needs of a hospital based supportive and palliative care caseload in conjunction with those of frailty.

Methods A retrospective case-note review of individuals referred to a hospital based supportive and palliative care team (HSPC) and frailty team (F) using standardised data collection sheet.

Results 200 case-notes were reviewed, 100 from each team. Individuals had broadly similar needs with the Clinical Frailty Scale (CFS) scoring 5 or above, 92\% from HSPC and $87 \%$ from frailty. The age differed unsurprisingly (74yrs vs. $84 \mathrm{yrs}$ (average)) with similar numbers of individuals having had polypharmacy (> 5 or more)(69\% vs. $71 \%)$. Significant differences were noted in the predominating diagnosis (cancer $-69 \%$ vs. $3 \%)$, multi-morbidity ( $41 \%$ vs. $67 \%$ ) and those residing in care homes (2\% vs. 19\%).

Conclusions The findings highlight broadly similar needs for individuals whether they are under a supportive and palliative care team or that of a frailty team within a hospital setting. In particular multi-morbidity, polypharmacy and needs as assessed by Clinical Frailty Score (CFS) were broad similar. The opportunity lies within healthcare providers using similar processes and pooling resources to meet the needs of frailty as well as those of supportive and palliative care. The use of a universal holistic assessment tool is a priority for further exploration within these teams and the individuals they care for. 